\title{
CÁNCER HEPÁTICO, PROBLEMA DE SALUD SUBESTIMADO
}

\section{LIVER CANCER, AN UNDERESTIMATED HEALTH PROBLEM}

\author{
Adriana Ramírez-Cosmes ${ }^{1}$ Gabriela Carrasco-Torres² José Fernando Sánchez-Pino ${ }^{1}$ \\ Irving Martínez-Contreras ${ }^{1}$ Rafael Baltiérrez-Hoyos³ y Verónica Rocío Vásquez-Garzón³
}

Fecha de recepción: 28 de noviembre de 2016

Fecha de aceptación: 8 de noviembre de 2017

\begin{abstract}
Resumen - El cáncer hepático es la segunda causa de muerte por cáncer más común a nivel mundial, el cuarto con mayor incidencia y el tercero más letal en México. Debido a los malos hábitos y la inadecuada alimentación de la población mexicana, ésta se encuentra en un riesgo elevado de desarrollar este tipo de enfermedad. En la actualidad, es difícil hacer un diagnóstico práctico y seguro del cáncer de hígado en etapas tardías y aún es más difícil en fases tempranas. Esta situación sumada a la escasa viabilidad y efectividad de los tratamientos, y a la carencia de registros fidedignos de incidencia nacional, anticipan una supervivencia muy baja. Es por eso que se requiere de una mayor atención y comprensión de dicho padecimiento, para la identificación de blancos terapéuticos y nuevos tratamientos oportunos. En este artículo revisamos los principales factores de riesgo del cáncer hepático, sus características biológicas, los actuales métodos de diagnóstico y las nuevas propuestas para su detección, permitiendo conocer el panorama contemporáneo y contribuir a investigaciones respecto de este problema de salud y reducir las altas tasas de incidencia y mortalidad.
\end{abstract}

\section{Palabras clave:}

Cáncer de hígado, factores de riesgo, biomarcadores, diagnóstico.
Abstract - Liver cancer is the second most common cancer death cause worldwide, the fourth with the highest incidence and the third most lethal in Mexico. Due to the bad habits and inadequate nutrition of the Mexican population, it is at a high risk of developing this type of disease. At present, it is difficult to make a practical and safe diagnosis of liver cancer in later stages and it is even more difficult in early stages. This situation, added to the scarce viability and effectiveness of the treatments, and the lack of reliable national incidence records, anticipate a very low survival rate. That is why it requires a greater attention and understanding of this condition, for the identification of therapeutic targets and new timely treatments. In this article we review the main risk factors for liver cancer, their biological characteristics, current diagnostic methods and new proposals for their detection, allowing us to know the contemporary panorama and contribute to research on this health problem and reduce the high rates of incidence and mortality.

\section{Keywords:}

Liver cancer, risk factors, biomarkers, diagnosis.

\footnotetext{
${ }^{1}$ Facultad de Medicina y Cirugía, Universidad Autónoma "Benito Juárez" de Oaxaca. Correo electrónico: adrii.raco@hotmail.com ${ }^{2}$ Escuela Superior de Medicina del Instituto Politécnico Nacional.

${ }^{3}$ Conacyt, Facultad de Medicina y Cirugía, Universidad Autónoma "Benito Juárez" de Oaxaca.

* Responsable Técnico. Correo electrónico: vrvasquezga@conacyt.mx
} 
E cáncer hepático es la segunda causa de muerte por cáncer más común a nivel mundial, con 746 mil defunciones al año, el cuarto con mayor incidencia y el tercero más letal en México. Este tipo de cáncer es un problema que avanza de manera exponencial desde 1970 a la fecha. México carece de registros fidedignos de casos nacionales de cáncer y sólo cuenta con cifras relacionadas con mortalidad; el dato más aproximado existente en la bibliografía médica es el del Globocan, donde se reporta la mortalidad en incremento en $400 \%$ en personas menores a 65 años (Globocan, 2012). Se prevé que aumentará de los 12.7 millones de nuevos casos de cáncer en 2008 a 21.4 millones en 2030; los países como México tendrán 75\% del acrecentamiento, siendo los cánceres de hígado relacionados con procesos infecciosos los de mayor recurrencia (Williams, 2014).

En un intento por identificar la incidencia en la población mexicana, en 1995 se creó el Registro Histopatológico de Neoplasias Malignas; sin embargo, ha quedado en un retraso importante en la recolección de datos. De acuerdo con la Secretaría de Salud, en el periodo 2000-2008 las enfermedades del hígado fueron la quinta causa de muerte a nivel nacional. Para Oaxaca, tumores malignos de hígado y de las vías biliares hepáticas ocuparon el tercer lugar y los padecimientos del hígado, incluyendo afecciones alcohólicas del hígado, ocuparon el quinto lugar (Secretaría de Salud, 2014).

\section{Factores de riesgo}

Existen diversos factores de riesgo que predisponen para el cáncer hepático. No obstante, éstos no lo indican todo, presentar uno o incluso varios no significa que se desarrollará la enfermedad (Kumar, Abbas y Aster, 2016).

Según Gomaa, Khan, Toledano, Waked y TaylorRobinson (2008), los principales factores de riesgo son:

- Infección causada por virus de hepatitis B y C

- Cirrosis por cualquier causa (principalmente alcoholismo)
- Malos hábitos alimenticios que pueden desencadenar hígado graso, el cual conlleva inflamación y cicatrización del hígado

- Consumo de aflatoxinas (toxinas producidas por hongos del género Aspergillus)

- Síndrome metabólico y enfermedades crónicodegenerativas de tipo vascular, como diabetes e hipertensión.

Los factores de menor peligro son:

- Cirrosis primaria de vías biliares

- Enfermedades por depósito de glucógeno

- Citrulinemia

- Porfiria cutánea tardía

- Tirosinemia hereditaria

- Enfermedad de Wilson

- Hemocromatosis hereditaria (Gomaa et al., 2008).

Se ha demostrado que una dieta equilibrada, rica en frutas y verduras, reduce el índice de cáncer (Liu, 2015). Sin embargo, un problema de gran importancia, que no se ha logrado contrarrestar y ha sido subestimado, es que la dieta de la población mexicana se caracteriza por ser alta en grasas trans, generadoras de radicales libres. Así también, en México el índice de pacientes con sobrepeso, diabetes mellitus y con enfermedades hepáticas crónicas es elevado; esto, junto con otros factores de riesgo existentes para desarrollar cáncer de hígado como el consumo excesivo de alcohol, hacen del diagnóstico oportuno una urgente prioridad de política pública, además de implementar las medidas necesarias para su prevención (Barquera, Campos-Nonato, Hernández-Barrera, Pedroza, RiveraDommarco, 2012), ya que actualmente cuando se diagnostica cáncer de hígado las posibilidades de tratamiento son escasas y poco efectivas.

En México sigue siendo una barrera importante el acceso a la información sobre este padecimiento, así como los procedimientos para su detección oportuna. Existen, además, diferencias importantes entre la cantidad y la calidad de la información disponible, 
aquella con la que cuenta la gente, y la manera en que la convierten en conocimiento y toman acciones en favor de su salud.

Si bien en este país, según datos de la Secretaría de Salud, hay más de 70 organizaciones de la sociedad civil (OSC) de lucha contra el cáncer, muchas de ellas operan bajo un enfoque meramente asistencial e informativo. Se requiere que un mayor número mejoren como sociedad civil organizada, de manera que impacten contundentemente la atención integral que se brinda a las personas con diagnóstico de cáncer de hígado.

\section{Manifestaciones clínicas}

Los síntomas son inespecíficos y se evidencian hasta que el paciente registra un deterioro importante de su salud, cuando presenta caquexia, debilidad y malestar general. La ascitis puede ser sanguinolenta, con posibilidad de que haya una trombosis portal, donde el cáncer ya es muy avanzado (Cornett y Dea, 2015; Plancarte, Guillén, Guajardo y Mayer, 2004). Existen otras manifestaciones, como fiebre de origen desconocido, ictericia asociada al cáncer hepático tales como una elevación de la fosfatasa alcalina y la GGT-Gammaglutamil transpeptidasa, sin incremento significativo de la bilirrubina, que constituyen lo que se conoce como patrón de colestasis disociada y se observa en la obstrucción intermitente de la vía biliar, granulomatosis hepática (Dufour, Lott, Nolte, Gretch, Koff y Seeff, 2000; Gopal y Rosen, 2000), hepatomegalia más dolor abdominal agudo e hipertransaminasemias en pacientes asintomáticos, pudiendo presentarse también una elevación del hemidiafragma derecho (Debs, Kassir, Amor, Martini, lannelli y Gugenheim, 2014).

Morfológicamente, se origina un cambio de tamaño que sucede en el espacio portal de las células hepáticas con un núcleo amorfo (figura 1). Los hepatocitos tienen un incremento ligero de hipercromatismo y/o pleomorfismo celular; además, las células que muestran cambio de tamaño suelen formar pequeños nódulos expansivos dentro de un solo lobulillo parenquimatoso. Los nódulos pueden ser con displasia de bajo y de alto grado (Sun y Song, 2015; Assy, Nasser, Djibre, Beniashvili, Elias y Zidan, 2009).

Macroscópicamente se puede visualizar:

- Una masa unifocal (habitualmente grande)

- Nódulos multifocales distribuidos de forma amplia y de tamaño variable

- Un cáncer infiltrante difuso

- Cambio de color del hígado (rojo pardo), dependiendo de la alteración a la que se asocie, de color blanquecino si es cirrótico o amarillento si es un hígado graso.

\section{Diagnóstico}

El diagnóstico se realiza mediante técnicas de imagenología y serológicas; sin embargo, la utilización de marcadores como prueba de diagnóstico, entre ellas alfa-fetoproteína, ha tenido deficiencias debido a que también se presenta en pacientes con hepatitis y cirrosis (Song, Xia, Inagaki, Hasegawa, Sakamoto, Kokudo y Tang, 2016).

Las pruebas suelen realizarse en enfermos considerados de alto riesgo:

1. Pacientes con cirrosis de cualquier causa

2. Pacientes infectados con el virus de hepatitis $B$ en cualquier etapa

3. Pacientes infectados con el virus de hepatitis C en fase cirrótica o con carga viral elevada.

4. Hepatopatías crónicas de cualquier etiología previamente estables con descompensación súbita inexplicable (Kumar et al., 2016).

Una vez detectada alguna lesión sospechosa se debe dar seguimiento cada seis a 12 meses, por medio de una tomografía axial computarizada contrastada de cuatro fases (simple, arterial, venosa y portal). Los tratamientos abarcan desde resección, trasplante hepático, inyección de alcohol, ablación por radiofrecuencia hasta quimioembolización (SMe0, 2012). 


\section{Biología celular asociada al cáncer hepático}

Se han identificado las características principales que adquieren las células cancerosas, como evasión de la apoptosis, señalización sostenida para crecimiento, insensibilidad a señales de anticrecimiento, potencial replicativo ilimitado, sostenida angiogénesis, así como invasión de tejido y metástasis; además de alteraciones genéticas asociadas a funciones toxicológicas, biológicas y canónicas. Las principales vías de señalización desreguladas son la del factor de crecimiento transformante/receptor del factor de crecimiento transformante (TGF-beta/TBR), factor de crecimiento parecido a insulina/receptor del IGF1 (IGF/IGF-R1) (figura 2), factor de crecimiento de hepatocitos (HGF/MET), Wnt/beta-catenina/FZD y el factor de crecimiento transformante/receptor del factor de crecimiento epidermal (TGF-alfa/EGFR) (Hanahan y Weinberg, 2000).

Los nuevos tratamientos contra el cáncer de hígado, como AG1024 y Gefitinib, bloquean a nivel del receptor para IGF-R1 (Yao, Liu, Sheng y Huang, 2011); algunas otras drogas son Rapamycin, CCl-779 y RAD001, que se encuentra en investigación (Zaytseva, Valentino, Gulhati y Evers, 2012). En estudios clínicos de fase II, Sorafenib demostró reducir la proliferación celular y la angiogénesis del tumor, además, ocasionó una reducción del tumor en $43 \%$ de los casos (Kitisin, Pishvaian, Johnson y Mishra, 2007).

\section{Biomarcadores en cáncer hepático}

Uno de los grandes desafíos para la medicina y la ciencia es la detección del cáncer hepático en etapas tempranas, para un tratamiento oportuno; empero, debido a la heterogeneidad tumoral y la diversidad de los factores de riesgo, los tratamientos resultan ineficaces, por ello es necesario profundizar en el conocimiento de todos los factores que participan en la iniciación, desarrollo y progresión del cáncer. Esto ha llevado a los investigadores a indagar y aplicar distintas tecnologías de nueva generación en busca de biomarcadores tempranos útiles para localizar y desarrollar tratamientos no invasivos del cáncer hepático y mejorar la calidad de vida de los pacientes. En la última década, en la práctica clínica se busca emplear herramientas como biomarcadores séricos y pruebas de imagen que no resulten incómodos o riesgosos para el diagnóstico precoz y vigilancia del cáncer hepático (Singal, Volk, Waljee, Salgia, Higgins, Rogers y Marrero, 2009).

Existen métodos de diagnóstico como los marcadores de antígenos tumorales, entre ellos el carbohidrato-125, carbohidrato 19-9 y antígeno carcino-embrionario combinado con antígeno histopatológico, pero estos métodos aún continúan siendo un tanto inespecíficos. Además, diferentes países siguen analizando biomarcadores séricos como alfa fetoproteina (AFP) para el seguimiento de cáncer hepático; así como éste, están AFP-L3, des-carboxyprothrombina (DCP), Dickkopf-1 (DKK1) y midkine (MDK), (Song et al., 2016), así también se reportan micro-ARN's que están siendo considerados como biomarcadores, tales como miR-25, miR-375 y let-7f, los cuales podrían proporcionar una sensibilidad de $97,9 \%$ y una especificidad de $99,1 \%$ en el diagnóstico de cáncer hepatocelular (Li, Hu, Zhou, Chen, Liu, Zhang, Shen, Zhang y Zen, 2010).

Se ha demostrado la sobreexpresión a nivel génico y proteico de Ezri (EZR) y Podocalyxin (PODXL), ambas proteínas asociadas a un mal pronóstico de varios tipos de cáncer debido a su participación en la invasión y migración tumoral, sugiriéndolas como posibles marcadores para predecir el cáncer hepatocelular (Flores, López, Vásquez-Garzón, y Villa-Treviño, 2015). Asimismo, han surgido nuevas aportaciones como la aplicación de múltiples terapias mediante la inhibición de cinasas, como el Sorafenib; sin embargo, dichas terapias parecen ser limitadas, pues sólo incrementan la esperanza de vida de los pacientes de ocho a 11 meses (Llovet, Ricci, Mazzaferro, Hilgard, et al., 2008). Aún se realiza una ecografía abdominal, pero tiene escasa sensibilidad y especificidad que han limitado el diagnóstico (Singal et al., 2009). 
Por lo anterior, con el actual avance tecnológico se ha comenzado a utilizar herramientas como la proteómica y la genómica, con las que se pretende estudiar y encontrar cambios funcionales en el proteoma humano originados por patologías o padecimientos como el cáncer, para así comprender y caracterizar los mecanismos moleculares relacionados con la tumorigénesis y después validar biomarcadores proteicos asociados a tal enfermedad (Alfaro, Sinha, Kislinger y Boutros, 2014).

Se han analizado perfiles de expresión de proteínas en algunos tipos de cáncer, como el hepatocelular, con la finalidad de encontrar péptidos específicos de tumor; sin embargo, esto se ha vuelto un desafío, en virtud de la complejidad del genoma y transcriptoma, así como de los mecanismos de regulación de la expresión, mutaciones y otras aberraciones que han dificultado dicho propósito (Alfaro et al., 2014). Ejemplo de lo anterior es que se identificaron mediante proteómica cuantitativa biomarcadores urinarios potenciales para este tipo de cáncer, los cuales fueron detectados en pacientes y comparados con personas sanas, proponiendo realizar investigaciones posteriores que reafirmen lo antes dicho y puedan ser propuestos como marcadores para el diagnóstico oportuno (Shyh y King, 2016).

Siguiendo el empleo de técnicas moleculares, en los últimos años diversas investigaciones se han centrado en estudiar los micro ARN's y su participación en el cáncer. Estos son pequeñas secuencias de nucleótidos (19-20 nucleótidos) cuya función es regular la expresión genética al disminuir o bloquear la síntesis de proteínas (Maitri, Ferrajoli, Sood, LópezBerestein y Calin, 2016), participando además en una gran variedad de procesos biológicos, como diferenciación celular, proliferación y apoptosis, siendo más de un tercio de genes en humanos objetivo de dichas secuencias (Tomimaru, Eguchi, Nagano, Wada, Kobayashi, Marubashi et al., 2012) (figura 3). La desregulación de los micro-ARN's se ha relacionado con el desarrollo de algunas enfermedades (Wang, Ding, Li, Liu, Wu, Wu y Yu, 2016).
Hasta la fecha se han propuesto algunos micro-ARN's circulantes como posibles marcadores tumorales en distintos tipos de cáncer, como el de hígado, de pulmón, de mama y de tiroides; también se ha demostrado que distintos microARN's intervienen en diversas funciones y participan en el desarrollo de la tumorigénesis, asociando a muchos de éstos al microambiente tumoral, procesos intercelulares y respuesta inmune (miR-1792, miR-222, miR-339) (Maitri et al., 2016).

De lo anterior se cuenta con varias evidencias, en las que han evaluado la presencia de distintos micro ARN's que podrían estar implicados en el desarrollo de cáncer hepático. Se ha propuesto la combinación de miR-15b y miR-130 como un marcador útil para el diagnóstico de dicho cáncer (Liu, Yao, Wang, Wong, Lee, Fan, Poon, Gao y Luk, 2012). Asimismo, mediante qRTPCR demostraron que los niveles de micro-ARN-21 en plasma disminuyeron en pacientes con cáncer hepático después de realizase cirugía de resección. Sus estudios indican que los niveles de dicho microARN en plasma fueron mayores en 126 pacientes con cáncer hepático que en aquellos con hepatitis crónica y voluntarios sanos (Tomimaru et al., 2012).

A través de un microarreglo de expresión se encontró una expresión anormal de 92 micro-ARN's en tejidos tumorales en comparación con tejidos no tumorales adyacentes. De éstos, se observó una disminución de la expresión de miR-15b-5p, miR-338-5p y miR764 en el plasma postoperatorio, correlacionándose positivamente este último con el tamaño del tumor. Así, se propusieron estos tres micro-ARN's como posibles biomarcadores para el diagnóstico de cáncer de hígado (Chen, Chen, Liu, Li y Huang, 2015).

La expresión de diversos micro-ARN's no sólo está relacionada con una función oncogénica, pues hay algunas evidencias que catalogan a algunos como supresores de tumor; un ejemplo es el MiR-139$5 p$, reportado con disminución en su expresión en cáncer hepatocelular, esta baja expresión en tejidos cancerosos se ha vinculado con un mal pronóstico (Wang et al., 2016). 
A partir de tales evidencias y conociendo la capacidad de regulación de los micro- ARN's y otras pequeñas secuencias de ARN se han propuesto terapias dirigidas al silenciamiento de genes claves, 0 al silenciamiento de los propios micro-ARN's para mejorar la detección oportuna, evitar y reducir las posibilidades del desarrollo de cáncer de hígado (Sun, Zhang, Feng, Liu, Xie, Qin, Zhao y Wan, 2017; Yang, Zhao, Hou, Li, Wang, Wu, Sun, Han, Sun, Song, Huang y Shao, 2017; Li et al., 2015; Yang, Li, Chang, Wang, Song, Gao, Hu, Li, Liu, Yao y Huang, 2014).

\section{Conclusión}

En México, el cáncer de hígado ocupa uno de los primeros lugares de incidencia, además de ser una de las principales causas de muerte. Se plantea reafirmar el compromiso de contribuir al proceso de toma de decisiones, de formular e implementar proyectos, de fortalecer las instituciones y de ampliar la investigación sobre el cáncer de hígado como una prioridad en el tema de salud de mujeres, hombres y para los sistemas en la materia.
Hoy en día no existen biomarcadores de diagnósticos prácticos y confiables en etapas tardías y menos aún en tempranas, además de la escasa viabilidad y efectividad de los tratamientos, lo que repercute en una mayor tasa de mortalidad. Por lo tanto, la detección oportuna desempeña un papel crucial para un mayor éxito del tratamiento, o mejor todavía, la prevención del cáncer hepático solucionaría una de las causas de muerte más importantes por cáncer. De ahí que el estudio de las fases tempranas de la carcinogénesis ha tenido gran importancia para entender mejor el origen y progresión de dicha enfermedad, así como para encontrar métodos de diagnóstico efectivos. El estudio de la biología celular del cáncer nos dará un mejor panorama para comprender los procesos biológicos alterados durante esta enfermedad, así como ofrecer a la población mayor información y bases para la búsqueda de un método de diagnóstico efectivo y viable, así como posibles blancos terapéuticos.

\section{Figura 1.}

Tinción de Hematoxilina y Eosina. Biopsia de carcinoma hepatocelular. Se puede apreciar un carcinoma in situ, así como infiltrados inflamatorios, además de células binucleadas y núcleos amorfos a causa de la patología. A) $4 \mathrm{X}$ y B) $8 \mathrm{X}$.
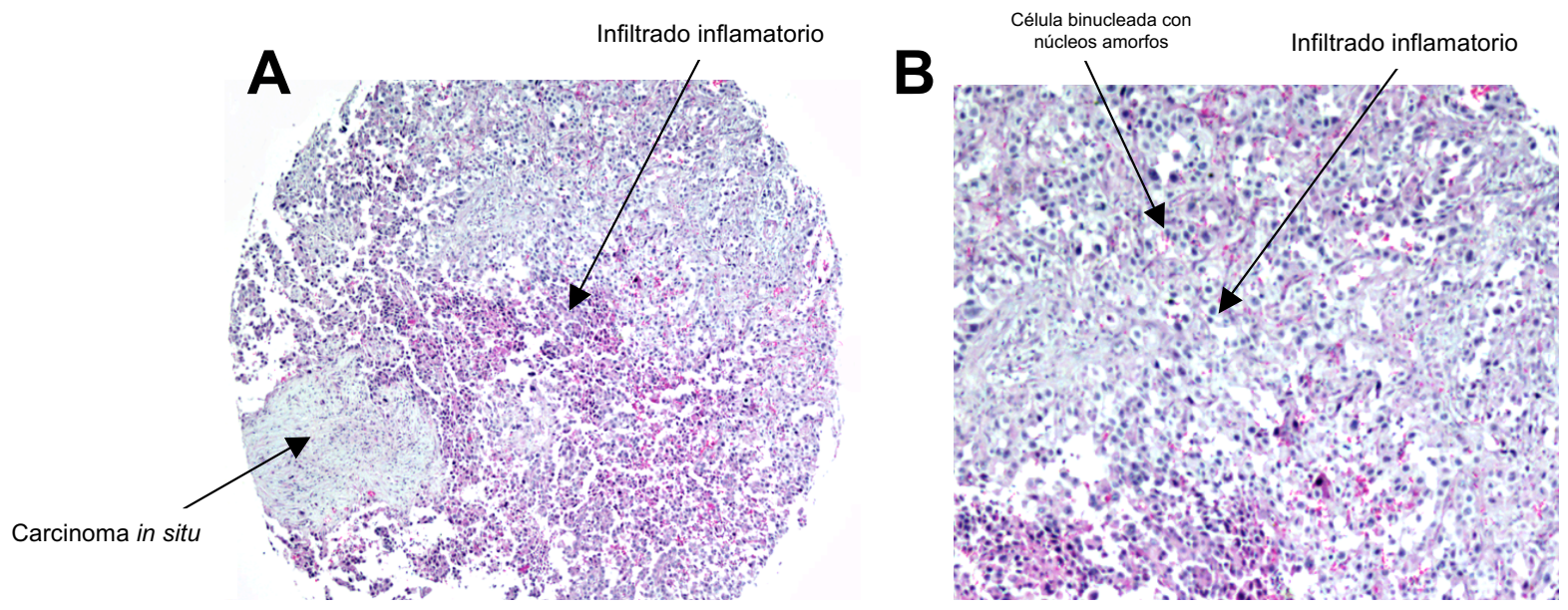


\section{Figura 2.}

Vía de señalización asociada a IGF. IGF-I e IGF-II se unen a su receptor (IGFR), un receptor tirosina quinasa (RTK) con alta afinidad que fosforila a las proteínas intracelulares, incluyendo a sustratos receptores de insulina (IRS), como PI3K, AKT/PKB y MAPK, las cuales participan en la inhibición de la muerte celular programada y activan la proliferación y crecimiento celular. AG1024 y Gefitinib bloquean a nivel del receptor para IGF (IGF-R1), Rapamicyn, CCI-779 y RAD001 y Sorafenib disminuyen la proliferación celular.



\section{Figura 3.}

Biogénesis de micro-ARN's. Se muestra la síntesis, procesamiento y maduración de micro-ARN's.

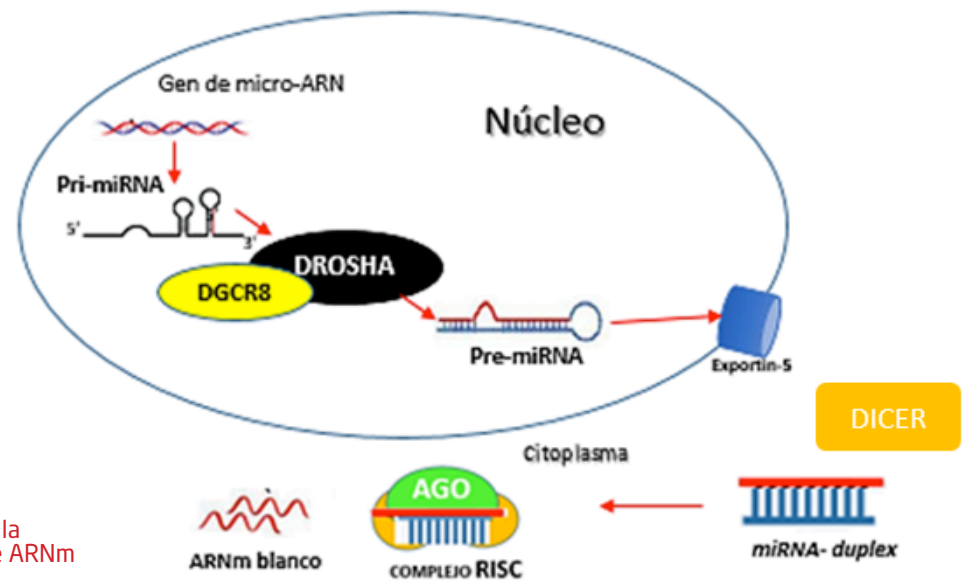




\section{Referencias}

Alfaro, J. A., Sinha, A., Kislinger, T. y Boutros, P. C. (2014). Onco-proteogenomics: cancer proteomics joins forces with genomics. Nat Methods, 11, $1107 \mathrm{e} 13$.

Assy, N., Nasser, G., Djibre, A., Beniashvili, Z., Elias, S. y Zidan, J. (2009).Características de las lesiones hepáticas sólidas comunes y recomendaciones para el diagnóstico. World J Gastroenterol., 15(26), 32173227.

Barquera, S., Campos-Nonato, I., Hernández-Barrera, L., Pedroza, A. y Rivera-Dommarco, J. A. (2012). Prevalencia de obesidad en adultos mexicanos. ENSANUT, 55(suppl), S151-S160.

Chen, Y. Chen, J., Liu, Y., Li, S. y Huang, P. (2015). Plasma miR-15b-5p, miR-338-5p and miR-764 as Biomarkers for Hepatocellular Carcinoma. Med Sci Monit., 21, 1864-1871.

Cornett, P. A. y Dea, T. O. (2015). Diagnóstico clínico y tratamiento. 54 ed. Mcgraw-Hill/Interamericana editores, S.A. de C.V.

Debs, T., Kassir, R., Amor, I. B., Martini, F., lannelli, A. y Gugenheim, J. (2014). Solitary fibrous tumor of the liver: report of two cases and review of the literature. Nt J Surg, 12(12), 1291-1294.

Dufour, D. R., Lott, J. A., Nolte, F. S., Gretch, D. R., Koff, R. S. y Seeff, L. B. (2000). Diagnosis and monitoring of hepatic injury. I. Performance characteristics of laboratory tests. Lin Chem, 46(12), 2027-2049.

Flores-Téllez, T. N. J., López, T. V., Vásquez-Garzón, V. R. y Villa-Treviño, S. (2015). Co-Expression of EzrinCLIC5-Podocalyxin is associated with migration and invasiveness in hepatocellular carcinoma. PLOS ONE, 10(7), e0131605.
Globocan. (2012). (IARC) Section of Cancer Surveillance.

Gomaa, A. I., Khan, S. A., Toledano, M. B., Waked, I. y Taylor-Robinson, S. D. (2008). Hepatocellular carcinoma: epidemiology, risk factors and pathogenesis. World J Gastroenterol, 14(27), 4300-4308.

Gopal, D. V. y Rosen, H. R. (2000). Abnormal findings on liver function tests. Postgrad Med., 107(2), 100-114.

Hanahan, D. y Weinberg, R. A. (2000). The hallmarks of cancer. Cell., 100(1), 57-70.

Kitisin, K1, Pishvaian, M. J., Johnson, L. B. y Mishra, L. (2007). Liver stem cells and molecular signaling pathways in hepatocellular carcinoma. Gastrointest Cancer Res., 1(4 Suppl 2), S13-21.

Kumar, V., Abbas, A. y Aster, J. (2016). Patología estructural y funcional. 9 ed. Barcelona: Elsevier.

Li, L. M., Hu, Z. B., Zhou, Z. X., Chen, X., Liu, F. Y., Zhang, J. F., Shen, H. B., Zhang, C. Y. y Zen, K. (2010). Serum microRNA profiles serve as novel biomarkers for HBV infection and diagnosis of HBV-positive hepatocarcinoma. Cancer Res., 70, 9798-9807.

Li, S., Li, J., Fei, B. Y., Shao, D., Pan, Y., Mo, Z. H., Sun, B. Z, Zhang, D., Zheng, X., Zhang M., Zhang, X. W. y Chen, L. (2015). MiR-27a promotes hepatocellular carcinoma cell proliferation through suppression of its target gene peroxisome proliferator-activated receptor $\otimes$. Chin Med J., 128(7), 941-947.

Liu, A. M., Yao, T. J., Wang, W., Wong, K. F., Lee, N. P., Fan, S. T., Poon, R. T., Gao, C. y Luk, J. M. (2012). Circulating miR-15b and miR-130b in serum as potential markers for detecting hepatocellular carcinoma: a retrospective cohort study. BMJ Open, 2(2), e000825. 
Liu, H. (2015). Fruit and vegetable consumption and risk of bladder cancer: an updated metaanalysis of observational studies. Eur J Cancer Prev, 24, 508-516.

Llovet, J. M., Ricci, S., Mazzaferro, V., Hilgard, P., Gane, E., Blanc, J. F., De Oliveira, A. C., Santoro, A., Raoul, J. L., Forner, A., Schwartz, M., Porta, C., Zeuzem, S., Bolondi, L., Greten, T. F., Galle, P. R., Seitz, J. F., Borbath, I., Häussinger, D., Giannaris, T., Shan, M., Moscovici, M., Voliotis, D. y Bruix, J. (2008). Sorafenib in advanced hepatocellular carcinoma. N Engl J Med., 359, 378e90.

Maitri, Y., Ferrajoli, A., Sood, A. K., López-Berestein, G. y Calin, G. (2016). MicroRNA Therapeutics in Canceran Emerging Concept. EBioMedicine, 12, 34-42.

Plancarte, R., Guillén, M. R., Guajardo, J. y Mayer, F. (2004). Ascites in cancer patients. Physiopathology and therapeutic options. Rev Soc Esp Dolor, 11, 156-162.

Secretaría de Salud. 2014. Mortalidad. Dirección general de información en salud. México, D.F.

Shyh-Horng, C. y King-Teh, L. (2016). Proteomic analysis and translational perspective of hepatocellular carcinoma: Identification of diagnostic protein biomarkers by an onco-proteogenomics approach. Elsevier, 32(11), 535-544.

Singal, A., Volk, M. L., Waljee, A., Salgia, R., Higgins, P., Rogers, M. A. y Marrero, J. A. (2009). Meta-analysis: surveillance with ultrasound for earlystage hepatocellular carcinoma in patients with cirrhosis. Aliment Pharmacol Ther., 30, 37-47.

SMeO. (2012). Guía mexicana de tratamiento del hepatocarcinoma avanzado. Gaceta Mexicana de Oncología, 11, Supl. 2.

Song, P. P., Xia, J. F., Inagaki, Y., Hasegawa, K., Sakamoto, Y., Kokudo, N. y Tang, W. (2016). Controversies regarding and perspectives on clinical utility of biomarkers in hepatocellular carcinoma. World J Gastroenterol, 22, 262-274.

Sun, G., Zhang, C., Feng, M., Liu, W., Xie, H., Qin, Q., Zhao, E. y Wan, L. (2017). Methylation analysis of p16, SLIT2, SCARA5, and Runx3 genes in hepatocellular carcinoma. Medicine, 96(41), e8279.
Sun, H. y Song, T. (2015). Hepatocellular carcinoma: advances in diagnostic imaging. Drug Discov Ther, 9, 310-318.

Tomimaru, Y., Eguchi, H., Nagano, H., Wada, H., Kobayashi, S., Marubashi, S., et al. (2012). Circulating microRNA-21 as a novel biomarker for hepatocellular carcinoma. Journal of Hepatology, 56, 167-175.

Wang, Z., Ding, Q., Li, Y., Liu, Q., Wu, W., Wu, L. y Yu, H. (2016). Reanalysis of microRNA expression profiles identifies novel biomarkers for hepatocellular carcinoma prognosis. Tumour Biol., 37(11), 1-9.

Williams, R. (2014). Addressing liver disease in the UK: a blueprint for attaining excellence in health care and reducing premature mortality from lifestyle issues of excess consumption of alcohol, obesity and viral hepatitis. Lancet, 384(9958), 1953-1997.

Yao, W. F., Liu, J. W., Sheng, G. L. y Huang, D. S. (2011). Blockade of IGF-IR exerts anticancer effects in hepatocellular carcinoma. Mol Med Rep., 4(4), 719-722.

Yang, Y., Li, M., Chang, S., Wang, L., Song, T., Gao, L., Hu, L., Li, Z., Liu, L., Yao, J. y Huang, C. (2014). MicroRNA-195 acts as a tumor suppressor by directly targeting Wnt3a in HepG2 hepatocellular carcinoma cells. Mol Med Rep., 10(5), 2643-2648.

Yang, Y., Zhao, Z., Hou, N., Li, Y., Wang, X., Wu, F., Sun, R., Han, J., Sun, H., Song, T., Huang, C. y Shao, Y. (2017). MicroRNA 214 targets Wnt3a to suppress liver cáncer cell proliferation. Mol Med Rep., 16(5), 6920-6927.

Zaytseva, Y. Y., Valentino, J. D., Gulhati, P. y Evers, B. M. (2012). MTOR inhibitors in cancer therapy. Cancer Lett, 319, 1-7. 


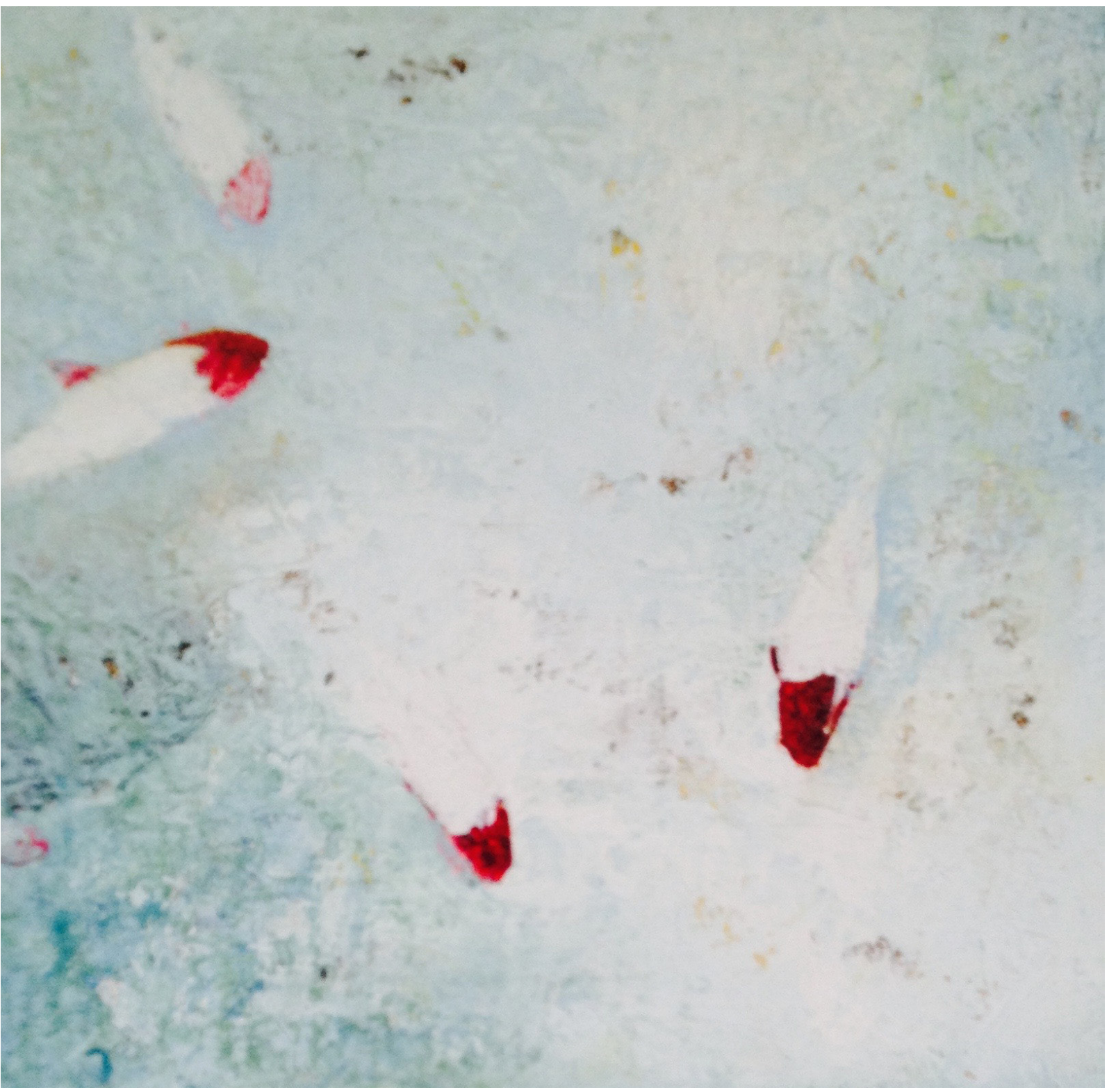

Melisa Sánchez Méndez.

"Serie de peces II",

encausto/tela,

$40 \times 40 \mathrm{~cm}$

2016. 